\title{
Hydrogen Influence on Gallium Arsenide Thin Films Prepared by RF-Magnetron Sputtering Technique
}

\author{
J. Vilcarromero ${ }^{1}$, R. Bustamante ${ }^{1}$, and J.H.D. da Silva ${ }^{2}$ \\ ${ }^{1}$ Grupo de Novos Materiais, Instituto de Pesquisa e Desenvolvimento, \\ Universidade do Vale do Paraíba, São José dos Campos - SP, Brazil \\ ${ }^{2}$ Departamento de Física, Faculdade de Ciências, Universidade Estadual Paulista, Bauru - SP, Brazil
}

Received on 8 December, 2005

\begin{abstract}
We investigate the effect of the hydrogen intentional incorporation on the structural properties of the amorphous gallium arsenide prepared by rf-magnetron sputtering technique. The properties of the non-hydrogenated films are: band gap of $1.4 \mathrm{eV}\left(\mathrm{E}_{04}\right)$, Urbach energy of $110 \mathrm{meV}$, stoichiometric composition ([As]/[Ga] = 0.50), and dark conductivity of about $3.2 \times 10^{-5}(\Omega . \mathrm{cm})^{-1}$. Hydrogen was incorporated in the films by the introduction of an electronically controlled $\mathrm{H}_{2}$ flux during deposition, keeping constant the other deposition parameters. It was observed that small hydrogen incorporation produces a great change in the structural properties of the films. The main changes result from the formation of GaAs nanocrystals with mean sizes of about $7 \mathrm{~nm}$ into the amorphous network.
\end{abstract}

Keywords: Hydrogen; Gallium arsenide; RF-magnetron sputtering

\section{INTRODUCTION}

In the last decade the study on the amorphous GaAs indicated that this material reached properties comparable with the other two widely studied amorphous materials, a-Si:H and a-Ge:H [1-5]. Recent studies, reported by Campomanes et al. [1], using flash evaporation, and older studies by Murri et al. [2] and Seguin et al. [5] using reactive sputtering, show that it is viable to get amorphous GaAs and GaAs:H with good optical and electronic quality. In the literature related to the tetrahedrally bonded amorphous semiconductors it is known that the presence of hydrogen can improve of considerably the electronic properties of these materials [2-5]. Among the improved properties are the decrease of the Urbach energy parameter, lattice stress relief, and more ordered local atomic structure [4-8].

Previous studies also have shown that is possible to produce nanocrystalline GaAs, using processes that involve [8] or not [9] hydrogen incorporation. The new properties of the nanostructured GaAs open new possibilities of technological applications for this widely used electronic material. In this work we present the effect of the intentional hydrogen incorporation in the structural properties of amorphous and nanocrystalline GaAs films prepared by rf-magnetron sputtering.

\section{MATERIAL AND METHODS}

The rf-magnetron sputtering deposition technique was used in order to prepare hydrogenated and non-hydrogenated GaAs. Silicon, 7059 Corning glass, and quartz substrates were used in each deposition to allow the different characterizations carried through on these thin films. In this work we present the studies on morphology and structural properties.

The rf-magnetron sputtering deposition system consists of an ultra-high vacuum deposition chamber (Kurt J. LeskerSystem I). A GaAs target (3 inch diameter, $99.99 \%$ purity), was used in the depositions. The substrates were kept at fixed distance $(5 \mathrm{~cm})$ from the target in a planar configuration. The vacuum was attained using a rotary vane pump (Edwards E2M28) in series with a turbo molecular pump (1 $\mathrm{m}^{3} / \mathrm{min}$ Seiko Seiki). The residual pressure in the system was better than $1.0 \times 10^{-6}$ mbar before each deposition. The Ar and $\mathrm{H}_{2}$ flow rates in the system were controlled using electronic (MKS) mass flow controllers.

The total working pressure in the deposition chamber was controlled using a capacitive active gauge controller and a mass flow controller (BOC-Edwards-Model 1501). The radio frequency generating system works in $13.56 \mathrm{MHz}$, and can provide RF power up to $600 \mathrm{~W}$. An impedance matching box (Advanced Energy rfx-600) was used to optimize the relation of effective / reflected power. The control of substrate temperature was made by a Neocera system. This system allows the control of the temperature from ambient up to $800 \circ \mathrm{C}$.

The analysis of the residual gases was carried out using a quadruple mass analyser (Acquad model ECU 9313). Approximately $60 \%$ of the residual contaminants correspond to water. After the residual gas analyses, the argon (99.9999\% purity) introduced in the chamber. The argon flow rate was changed as hydrogen flux was introduced into the chamber in order to remain constant the total pressure.

The starting parameters for hydrogenated samples were chosen after optimization of optical and electronic quality of non-hydrogenated samples: rf-power of $30 \mathrm{~W}$, total pressure of $2,0 \times 10^{-3}$ mbar, and room temperature substrate. The hydrogen flow introduced in the deposition chamber was varied from 0 up to $36 \mathrm{sccm}$.

The samples were characterized using Fourier transform infra-red transmission spectroscopy, micro-Raman technique and X-rays diffraction Spectroscopy.

The Fourier transform infra-red transmission (FTIR) spectra were carried through in the region of 200 to $4000 \mathrm{~cm}^{-1}$ using a Bio-Rad spectrometer. The Raman scattering spectra were obtained using the Argon laser green line (514.5nm). Ten 1 minute scans were acquired in each spectrum using a Jobin Ivon T64000 spectrometer with a CCD system. The X- 
rays diffraction measurements had been carried through in a Rigaku Ultima $2000^{+}$system. The scans were made in the $\theta-2 \theta$ configuration, in the $20.0-60.0$ range, with a 0.02 step, with the $\mathrm{Cu}-\mathrm{K} \alpha$ radiation. The composition of the films was obtained using a scanning electron microscope connected to an electronic dispersion spectrometer of (EDS). Measures of EDS had been carried through with energy of $10 \mathrm{KV}$ in Joel model 5310 equipment. The precision of the determination is of the order of 1 at.\%. The equipment is sensible to the light elements also to carbon.

\section{RESULTS AND DISCUSSION}

The sample composition, measured with EDS, show that the hydrogenated a-GaAs samples are off-stoichiometric ([As]/[Ga] $=0.50)$. The increase of the hydrogen flux in the deposition chamber produced an As excess of the order of 2 at.\% above stoichiometry. Fig. 1 shows the FT-infrared transmission spectra of the a-GaAs samples in which the flow of hydrogen was the only changed parameter.

Some infrared absorption bands (Fig. 1), attributed to the $\mathrm{Ga}-\mathrm{H}$ bond vibrations and to the GaAs phonon modes [6], can be clearly observed. The bands located around $1870 \mathrm{~cm}^{-1}$, $1750 \mathrm{~cm}^{-1}$ and $700 \mathrm{~cm}^{-1}$ can be attributed [6] respectively to the $\mathrm{Ga}-\mathrm{H}_{2}$ stretching, Ga-H stretching, and $\mathrm{Ga}-\mathrm{H}_{2}$ bending modes, while the bands at $250 \mathrm{~cm}^{-1}$ and $520 \mathrm{~cm}^{-1}$ are related respectively to the TO and $2 \mathrm{TO}$ GaAs phonons. The absorption at $1100 \mathrm{~cm}^{-1}$ is due to the $\mathrm{Si}$ substrate. The only band that could possibly be attributed to the As-H (wag) mode is located at $\sim 530 \mathrm{~cm}^{-1}$, and is too close to the $2 \mathrm{TO}-\mathrm{GaAs}$ to be resolved. The bands at $2130 \mathrm{~cm}^{-1}$ and $2040 \mathrm{~cm}^{-1}$ expected respectively for the As- $\mathrm{H}_{2}$ and As-H bonds do not appear in the spectrum. The sprouting of some absorption bands attributed to the $\mathrm{Ga}-\mathrm{H}$ bonds is a confirmation that the hydrogen is effectively introduced in the amorphous network. This observation is probably related to the shifts observed in the conduction and valence band edges of amorphous and microcrystalline GaAs [7], which cause optical gap opening and Urbach energy decrease $[2,8]$. These effects are indicative of the decrease in disorder and in density of states inside the pseudo-gap $[2,8]$.

Figure 2 shows the micro-Raman scattering spectra of the GaAs films for different hydrogen flows in the deposition chamber. We can observe that the samples without hydrogen present two broad bands in $60 \mathrm{~cm}^{-1}$ and $230 \mathrm{~cm}^{-1}$. These bands are respectively characteristic of the acoustic and optical vibration modes of the amorphous GaAs. When the hydrogen flow is increased the $230 \mathrm{~cm}^{-1}$ band displays a narrowing and a more structured line shape. Two small subbands are now apparent. These sub-bands can be associated to the LO and TO vibration modes of crystalline GaAs, although they are broadened and their frequencies being shifted to lower values due to the disorder in the network. This effect is indicative of the presence GaAs nanocrystals in the material. The formation of nanocrystals when smaller hydrogen amounts are introduced makes clear the importance of hydrogen in the process. The nanocrystal formation was also observed by Campomanes et al. [1] in non-hydrogenated GaAs

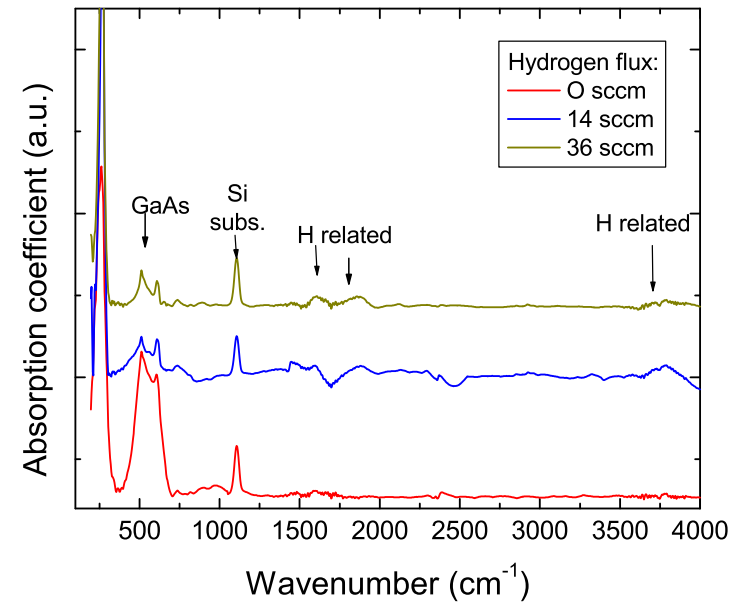

FIG. 1: FT-Infrared spectra of the hydrogenated GaAs samples as a function of hydrogen flux.

samples prepared by flash evaporation after thermal treatment at $600 \mathrm{C}$ for two hours. Feng and Zallen [9] also have produced a nanocrystalline GaAs structure. In this case the structure was attained by $\mathrm{Be}^{+}$ion bombardment of a crystalline GaAs surface.

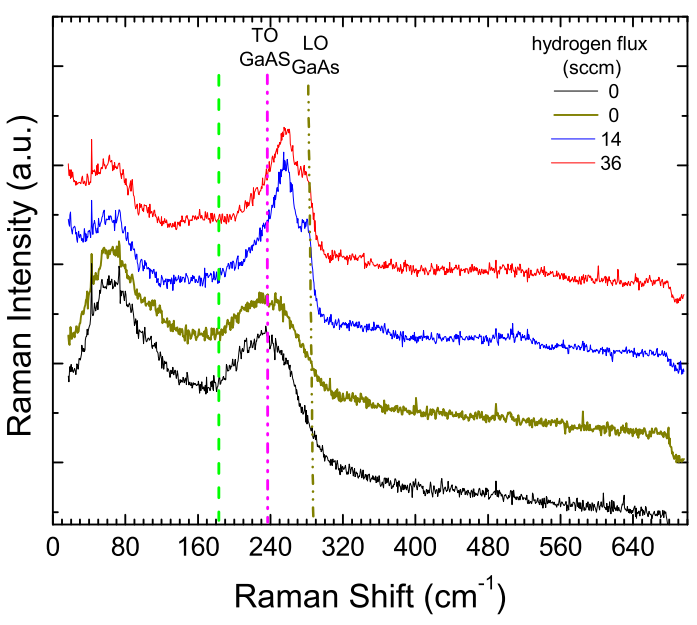

FIG. 2: Micro-Raman scattering spectra of hydrogenated GaAs films prepared under different hydrogen flux.

In order to better investigate the crystallization features observed in the micro-Raman analysis X-rays diffraction experiments were carried out. With this technique we can verify the existence or not of the nanocrystalline phase in the GaAs samples. In Figure 3 we present the X-ray diffraction patterns of the same set of samples, as function of the hydrogen 
flow through the deposition chamber. We can clearly observe the presence of three diffraction peaks attributed to the crystalline planes of the GaAs: 27.50 (GaAs (111)), 45.0 (GaAs (220)) and 54.0 (GaAs (311)). The sharp peaks present in the diffraction pattern of sample corresponding to a $36 \mathrm{sccm}$ hydrogen flux are due to the Si substrate. Using the GaAs peak widths and the Scherrers method we could estimate the crystallite sizes as being of the order of $7 \mathrm{~nm}$.

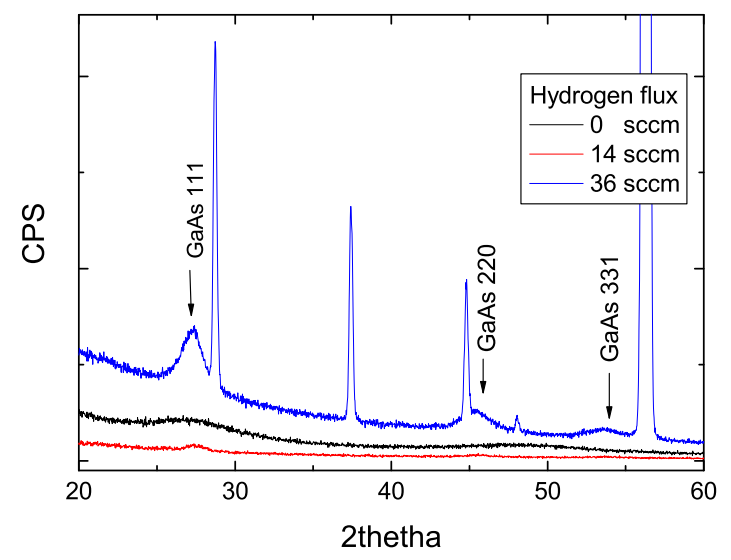

FIG. 3: XRD spectra of the hydrogenated GaAs samples prepared by rf-magnetron sputtering as a function o hydrogen flux.

Differently from our results, some previous works on hy- drogenation of GaAs have not shown evidences of crystallization due to hydrogen incorporation. Here, both microRaman and X-ray diffraction results indicate clearly that small amounts of hydrogen are producing big changes in the a-GaAs structure. This observation is probably related to the different structure condition of the a-GaAs used as starting point for hydrogen incorporation.

More complete studies about the hydrogenated nanocrystalline GaAs material are still needed in order to improve understanding of how hydrogen influence in the structural properties of a-GaAs, and how these films can reach optimized properties.

\section{CONCLUSION}

The effect of the hydrogen incorporation in GaAs films prepared by reactive magnetron sputtering was presented. The incorporation of small amounts of hydrogen to the plasma induces the presence of $\mathrm{Ga}-\mathrm{H}$ bonds, and of GaAs nanocrystals in the amorphous matrix, the crystallite sizes being of the order of $7 \mathrm{~nm}$.

\section{Acknowledgements}

The authors thank the Unesp - Bauru, Physics Department for the access to the X-ray facility, and Mrs. Lucia Maria, member of the INPE research staff, for the EDS measurements. The research was supported by FAPESP.
[1] R. R. Campomanes, J.H.D. da Silva, J. Vilcarromero, and L.P. Cardoso, Journal Non-Crystalline Solids, 238, 788 (2001).

[2] R. Murri, N. Pinto, L. Schiavulli, R. Fukuhisa, C. De Blasi, and D. Manno. Journal Non-Crystalline Solids, 151, 253 (1992).

[3] A. Carbone, F. Demichelis, and G. Kaniadakis. Il Nuovo Cimento, D 13, 571 (1991).

[4] V. Manorama, P.M. Dighe, and S. V. Bhoraskar. Appl. Phys. J. 68, 581 (1990).

[5] J.-L. Seguin, B. El Hadadi, H. Carchano, and K. Aguir, Journal
Non-Crystalline Solids, 238, 253 (1998)

[6] Z.P. Wang, L.Ley, and M. Cardona, Phys. Rev. B, 26, 3249 (1982).

[7] R. Karcher, Z.P. Wang, and L. Ley, Journal Of Non-Crystalline Solids, 59-60, 629 (1983).

[8] G.M. Azevedo, J.H.D. da Silva, and E. Avendao, Nuclear Instruments and Methods B, 238, 329 (2005).

[9] G.F. Feng and R. Zallen, Phys. Rev. B, 40, 1064 (1989). 